\title{
Internet Users' Attitudes Towards Online Targeted Advertisements
}

\author{
Mustafa Sait Yıldırım \\ Anadolu University, Eskişehir, Turkey
}

\begin{abstract}
Users leave their footprints about their interests while they are surfing on the Internet. Companies have the opportunity of capturing user's implicit data on the Internet by behavioral targeting. So that they can deliver the advertisements of the products or services that appeals to those consumers. Users are being exposed to targeted advertisements according to their demographic characteristics or web site choices. Each online behavior gives a clue about the consumer's product interests for the advertisers. Targeted advertising services such as these are currently provided by many companies including Google and Amazon. There are differences between the users' level of awareness and attitudes towards targeted ads. In this study Internet users' attitudes and avoidance towards targeted ads were evaluated.
\end{abstract}

Keywords: advertising, attitude, targeted ads, behavioral targeting, Internet

\section{Introduction}

Advertisers have started to attach more interest on the interactive methods for the promotion of their products and services together with the prevalence of the Internet all around the world (Yaakop et al., 2012). However, this paves the way for the new development. Consumers now want to have more control and are picky about the messages that they would like to have (Curran, Graham, \& Temple, 2011, p. 26). Advertising professionals head for new techniques to minimize the avoiding behaviors of the consumers against the message bombardment. The advance technology gives way to store and track the majority of the data of the consumers for the institutions or businesses. Customers' mobile phone call, Internet browsing or TV show that they have watched can be useful for storing the data with respect to the personal interests (favorite movies, music, restaurants or books etc.) and contents and advertisements can be provided fit for these behaviors (Kurkovsky \& Harihar, 2006; WANG et al., 2009). This technique can be conducted both on individual basis and applied for the clusters for demographic targeting of the consumers. Advertisement network on the basis of the behavioral targeting are provided by the companies in particular to Google and Amazon (Zhang et al., 2010, p. 1416). These advertisements, which are referred with different names in the literature, are addressed as online behavioral advertising, targeted advertising or behavioral targeted advertising. The basic principle of such advertisements is to track the Internet users at a wide framework, and evaluate their traces and show the best advertisement to the users, fit for their behaviors. In simple terms, advertisement networks select and provide advertisement suitable for the content of the web page in any web page. This is called as contextual advertising (Ur et al., 2012, p. 1). The more complicated form is to provide tailored made advertisement to the

Mustafa Sait Yıldırım, Ph.D. candidate, Faculty of Coomunication Sciences, Yunus Emre Campus, Anadolu University. 
consumers by using of the data related to the demographic, geographic features or web browsing behaviors.

\section{Behavioral Targeting}

Behavioral targeting is one of the new generation techniques, of which importance is getting increased day by day in the advertisement sector and which is used to improve the efficiency of the promotions by the Internet advertisements. According to Wikipedia, BT uses information collected on an individual's web-browsing behavior, such as the pages they have visited or the searches they have made, to select which advertisements to display to that individual. Targeting marketing, is addressed as the general strategy used to reach the consumer by using these data and defining the characteristics shared by the consumers such as social, economic, age, sex or ethnical origin (Johnson \& Grier, 2015, p. 235). Behavioral targeting is one of the most attention grabbing methods among the online advertisement techniques. Behavioral targeting is used to target the best consumer for the advertisement provided to increase the effect of the online advertisement. Now, web search of the consumer can be easily protected and tracked by the browser and exposed to advertisements according to the consumer search behaviors. Social networks such as Facebook or search engines such as Google provide advertisements, suitable for the field of interest of the users by using the consumer generated data. Even if behavioral targeting yields positive results for the advertisers, there are some concerns in terms of the consumers.

For example, in the paper of Johnson (2013) titled as Targeted Advertising and Advertising Avoidance, it is concluded that consumers avoid of the advertisements targeted to them (Johnson, 2012, p. 140). The advertisers are not target consumer groups that they can get feedback for their messages (Farahat \& Bahiley, 2012, p. 111). The treatment effect for the targeted group should be higher than targeted group for behavioral targeting to be significant and valuable. Several studies show that online tracking systems are capable to comprehensively build the profiles of the customers' online field of interest and cover most of their online behaviors (Liu et al., 2013, p. 1). Behavioral targeting is used to provide advertisements by filtering some of them from the huge advertisement data base and suitable for the field of interest of the users by following the online behaviors of the consumers. For example, a customer may see car advertisements at his Facebook page or movie web page. The reason is that he has visited the car web pages before. Targeted advertisements are useful for the advertisers. It has been seen as an opportunity to provide tailored made advertisement for the customer and specify who interested in more to the advertisement. For that reason, advertisement networks started to spend much more money to the targeted advertisement in comparison to the general advertisements. Online behavioral targeting provides suitable advertisements to the field of interest of the consumer and let him stay away from the unrelated advertisements. In technical terms, there are different ways to operate this system. The simplest way is that an advertiser may set a definitive cookie to the device of the consumer and relate it with the browsing activities of the consumer (Ur et al., 2012, p. 2). It will be kept in that way since the technicality is not our field of interest. Several topics such as privacy, trust, sex differences, interactivity and refraining from the advertisement with respect to the advertisement have been the subjects of many academic researches. The method of proceeding of the same variables in the new advertisement media and advertisement application in these media have been studied.

There are two important factors related to the behavioral targeting. First of all, providing offers which are suitable for the needs of the users by providing user friendly advertisements. The second one is the discussions whether it violates the privacy or not, that in intervening the personal space of the consumer. The foundation of 
this research is the attitudes of the users towards these two factors. What do the Internet users in Turkey think about the behavioral targeting and targeted advertisements and at which level are their avoiding or positive attitudes? This study has been conducted to find answers to these questions.

\section{Methodology}

We recruited 21 participants for an interview. All participants were recruited from the students of Anadolu University, Turkey. We choosed the participants who had no degree or job in computer science or information technology. We conducted a semi-structural interview. Interview questions were adapted from Blasé Ur's (2012) research. Some questions were removed and some new ones were added to the interview in order to adopt the semi-structural interview suitably for the research. At this stage, two academics expert in the field of view is taken. In addition, because of the reliability of the interview questions, pilot interviews were conducted with four participants, and the questions in the form were tested in terms of clarity and understandability. Due care was given to prepare an interview environment in which the participants would feel comfortable and peaceful to reveal their viewpoints, and a proper interaction medium was prepared. Information on the study was given to the participants during the interviews. A recording device was used in order to prevent data loss during the interviews. The participants were informed about the recording device, and it was added that they could have some parts or the whole of the recording erased at the end of the interviews depending on their wishes.

The semi-structured interview form consisted of 2 parts and 9 items (except for the sub-questions). In the first part of the form, there were 3 items on the attitudes of the participants on Internet Advertisements. In the second part: Firstly, questions on the information levels of the participants about Targeted Advertisements were asked. Then, questions were asked about the benefits of the Targeted Advertisements for the Internet users and other stakeholders. The participants were also asked about their information on the access level of the Online Behavioral Targeting activities. In addition, they were also asked about their ideas on the disadvantages of the Online Behavioral Targeting. The interviews were conducted face to face. Written notes were taken during the interviews.

\section{Participants}

Twenty-one participants were included in the study: 10 of them were female, and 11 of them were male. All of the participants were chosen among the undergraduate students of Anadolu University, and their ages varied between 18 and 23. Special attention was given for the issue of selecting those students who had no history on Computer Sciences or Information Technologies. Since the number of the sampling was limited, it is not claimed that the participants represent the universe of the Internet users. Therefore, in this study, the purpose is not obtaining statistically significant results; but rather, the purpose is to focus on the attitudes of ordinary Internet users on targeted advertisements.

\section{Analysis}

The interviews were recorded with a voice recorder, and written notes were taken during the interviews. After the interviews, the recorded information was converted into text. In order to ensure the reliability of the information, two people from the field listened to the interviews and read the texts to test the agreement between the recordings and the text. 
After the interviews were completed, a table of codes consisting of forthcoming themes was formed. The raw data obtained from the interviews were encoded and the categories were defined. The data then were classified under these categories and made meaningful. The encoding and categorizing processes were performed in recurrent style. By doing so, the unnecessary encodings were eliminated sticking to the problem and to the purpose of the study, and new codes were added into parts where necessary. In the last stage, the findings were defined and evaluated. Also, some astonishing quotations, which reflect the viewpoints of the participants, were given.

\section{Results}

The general attitude of the participants on "Internet Advertising" can be evaluated as being unreliable. In addition, it was also determined that the awareness levels of the participants on the possibility of the Targeted Advertisement Networks accessing the personal information of the consumers were high. In this part, the attitudes of the consumers about the Internet Advertisements and Targeted Advertisements were given in titles.

\section{Attitudes Towards Internet Advertising}

When the attitudes of the participants towards Internet Advertisements are considered, it is possible to claim that they have a negative perception on this issue. 16 of the 21 participants gave answers to the question "What is the first thought that crosses your mind when you hear Internet Advertisement?" as follows; "They appear always, I am subjected to them even if I do not want, I cannot get rid of them". For example, 7th Participant said "When I hear Internet advertisement, first of all, I remember the advertisements that prevent me from watching the video I want to watch and annoy me. In addition, it is also annoying when another advertisement comes right in the middle of the video and I have to wait for 5-10 seconds to skip that advertisement". 14 of the participants answered as "No" to the question "Do you like Internet Advertisements?". 8 of the male participants used negative expressions about the Internet Advertisements. Based on this point, it is possible to claim that male participants have more negative attitudes towards such advertisements than the female participants. The number of the participants that answered as "Yes" to the question "Do you find Internet Advertisements useful?" is 11. Based on this finding it is possible to claim that although the first image that is formed in the minds of the consumers about Internet Advertisements is negative, they find them useful and beneficial. During the interview, the 1st Participant said "In fact, many Internet advertisements help us to reach the products we want. I find the Internet advertisements useful because we can reach the information on the properties, the price and how to purchase the products".

\section{Tailored Advertising}

The participants were asked the question "Do you think that the Internet Advertisements you are exposed on the Internet appear in accordance with your areas of interest?", and the majority of them answered that the advertisements on the Facebook were in accordance with their areas of interest. In addition, when they were asked whether the advertisements were useful for their areas of interest or not, it became obvious that 15 of the participants found the advertisements useful.

Again, the majority of the participants who had positive attitudes about the issue of encountering Internet Advertisements that were in accordance with their areas of interest consisted of females. 9 women participants said that they found these types of advertisements useful. The participants who did not find these types of Internet Advertisements useful showed the excessive exposure of the Internet Advertisements as their excuses. 
For example, the 4th Participant said "For once, I am exposed to Internet Advertisements on the product I search for, sometimes for days. Each time I enter Facebook I face the advertisements of a product that I searched for days before or of a product even I have already purchased". The participants were asked the question "What are your opinions on how the advertisement companies determine your areas of interest?", and 18 participants expressed similar opinions, stating that they were presented the Internet advertisements with the data captured from Google Search Engine. Some participants said that some of the questionnaires on the Internet were for archiving their information, and the companies made use of these questionnaires to reach their target customers.

For example, the 9th Participant said, "Before I watch news or a video, I am subjected to questionnaires having some questions on my gender, age, profession and areas of interest. They have information on me because I fill in these questionnaires". The 18th Participant said "We enter our information to Facebook, Twitter or Instagram accounts, and in addition, we give the information about ourselves even our identity number when we purchase something online, therefore, advertisement companies do not have difficulty in presenting the advertisements of their products targeted to us".

\section{Attitudes Towards Online Behavioral Targeting and Targeted Advertising}

First of all, Online Behavioral Targeting for all consumers in general have beneficial sides; however, they also have some drawbacks like obtaining personal information. In this study, it was observed that the concerns on privacy are dominant after considering the Behavioral Targeting and after thinking on the fact that the personal information is more available rather than the beneficial sides of this technique.

The majority of the participants are aware of the beneficial sides of the targeted advertisements for the advertisement companies. 17 participants said "Yes" to the question "Do you think that targeted advertisements are beneficial for advertisement companies, and in your opinion, what are their benefits?". 13 of the participants said that this advertisement type made it easier for the advertisement companies to reach their target audience; 4 of them said that it was a new source of income for the advertisers. The answer of the 6 th participant to this question is extremely comprehensive. "These types of advertisements are beneficial both for the consumers as well as the product/service providers. Think about it, how wonderful is it to know how to advertise and to know that the advertisement will not go in vain!"

All of the participants answered as "Yes" to the question "Do Behavioral Advertisements have negative sides?" The point on which all of the participants agree is the privacy issue. Access to private life and personal information are the clearest reasons for avoiding Behavioral Advertisements. 4 participants said that they found the idea of being watched or being followed annoying. The 12th participant said "While I am searching online, the idea of a company or someone knowing what I am looking for makes me feel annoyed. I do not find it correct when my data are obtained by people I do not know", and summarized the concerns of all participants.

As a result, when they were asked "What are your general viewpoints on targeted advertisements, and why?" 9 of the participants said that they found it beneficial, and gave answers showing that their attitudes about these advertisements were positive. 6 of the participants who expressed positive opinions on this question were female. 4 participants, on the other hand, said that these advertisements were unnecessary and disturbing, and they also stated that especially the idea of obtaining the search results by others was disturbing. 8 participants gave double-sided answers expressing that they found it sometimes useful and sometimes boring. The 5th Participant said "I can shorten the duration of my purchase with the help of these advertisements. In addition, I 
have the opportunity of comparing the prices" showing her positive attitude about the Targeted Advertisements. The 20th Participant said "I am afraid of searching on the Google because of these advertisements. When I search for a product or a service, I encounter these advertisements on every website I visit, and this makes me annoyed".

The 27th Participant said "Their obtaining my personal information and search records make me disturbed, but there are times when I find these advertisements useful, because I think seeing different alternatives and advertisements on my areas of interest is a useful opportunity" showing that his viewpoints were not unidirectional on this issue.

The majority of the participants think that the advertisers reach their various information they share online together with their browsing history. The question "In your opinion, which of your information is reached by online advertisement networks?" was asked to the participants, and 17 participants answered that the advertisement companies could reach the city they lived in, mail addresses, and genders; 3 participant said that they could also reach their telephone numbers; 1 participant said that they could reach even their names. 5 participants said that the abovementioned information could be reached by companies and in fact they had heard that the companies shared their information with other companies, and even they sold the information to each other.

\section{Discussion and Conclusions}

In this paper, the attitudes of the participants about Targeted Advertisements and their awareness levels on this advertisement technique were examined. It was observed that the participants were aware of the benefits of the behavioral targeting both for the consumers and for the companies. It is possible to claim that the issue of privacy is the only problem that poses a handicap for the full adoption of the Targeted Advertisements by consumers. The most distinct finding determined in this study is the fact that although consumers do not deny the benefits of the Targeted Advertisements, and even find them beneficial, they stay at a certain distance to these types of advertisements because of the privacy issues and because the companies obtain their personal information.

Most of the time, consumers find Internet Advertisements disturbing and over-persistent. Generally, not having the control over the Internet Advertisements is the basic issue that disturbs them. They especially stated that Behavioral Targeting was an advantageous method for companies. It was understood that most of the participants did not have much information on Behavioral Targeting techniques. The participants have limited information on how their areas of interest are detected, archived and how their personal information is obtained. It was observed that the female participants were more open-minded about being subjected to advertisements on their areas of interest than the male participants. It was observed that the group with higher-level privacy concerns about targeted advertisements was the males. Consumers have concerns about their information being obtained by companies for Targeted Advertisements, and they also complain about being over-exposed to these types of advertisements. As the last item, although the attitude about Targeted Advertisements is positive in general, privacy concerns damage it.

\section{References}

Curran, K., Graham, S., \& Temple, C. (2011). Advertising on Facebook. International Journal of E-Business Development, 1(1), 26-33.

Farahat, A., \& Bailey, M. C. (2012). How effective is targeted advertising? In 21st International Conference on World Wide Web (pp. 111-120), ACM. 
Johnson, G. D., \& Grier, S. A. (2011). Targeting without alienating: Multicultural advertising and the subtleties of targeted advertising. International Journal of Advertising, 30(2), 233-258.

Johnson, J. P. (2013). Targeted advertising and advertising avoidance. Rand Journal of Economics, 44(1), 128-144.

Kurkovsky, S., \& Harihar, K. (2006). Using ubiquitous com puting in interactive mobile marketing. Pers Ubiquit Comput, 10, 227-240.

Liu, B., Sheth, A., Weinsberg, U., Chandrashekar, J., \& Govin-dan, R. (2013). AdReveal: İmproving transparency into online targeted advertising. In Proceedings of the Twelfth ACM Workshop on Hot Topics in Networks (pp. 1-7).

Ur, B., Leon, P. G., Cranor, L. F., Shay, R., \& Wang, Y. (2012). Smart, useful, scary, creepy: Perceptions of online behavioral advertising. Proceedings of Symposium on Usable Privacy and Security (SOUPS).

WANG, X. J., YU, M., ZHANG, L., CAI, R., \& Ma, W. Y. (2009). Argo: Intelligent advertising by mining a user's interest from his photo collections. ADKDD'09. ACM. 18-26.

Yaakop, A. Y., Mohamed Anuar, M., \& Omar, K. (2012). A Liaw Consumers' perceptions and attitudes towards advertising on Facebook in Malaysia. In World Business and Economics Research Conference, Auckland, New Zealand.

YAN, J., LIU, N., WANG, G., ZHANG, W., JIANG, Y., \& CHEN, Z. (2009). How much can behavioral targeting help online advertising?. Proceedings of the 18th International Conference on World Wide Web (pp. 261-270).

Zhang, H., Guerrero, C., Wheatley, D., \& Lee, Y. S. (2010). Privacy issues and user attitudes towards targeted advertising: A focus group study. Proceedings of the Human Factors and Ergonomics Society 54th Annual Meeting (pp. 1416-1420). 